\title{
ON EXACTNESS OF THE COARSE SHAPE GROUP SEQUENCE
}

\author{
Nikola Koceić Bilan \\ University of Split, Croatia
}

\begin{abstract}
The coarse shape groups are recently introduced. Given a pointed pair $\left(X, X_{0}, x_{0}\right)$ and a $k \in \mathbb{N}$, the relative coarse shape group $\check{\pi}_{k}^{*}\left(X, X_{0}, x_{0}\right)$, having the standard relative shape group $\check{\pi}_{k}\left(X, X_{0}, x_{0}\right)$ for its subgroup, is defined. They establish a functorial relations of the topological, homotopy and (coarse) shape category to the category of groups. Therefore, the coarse shape groups are new algebraic topological, homotopy and (coarse) shape type invariants. For every pointed pair of metric compacta $\left(X, X_{0}, x_{0}\right)$ and for every $k>1$, the boundary homomorphism $\partial_{k}^{*}: \check{\pi}_{k}^{*}\left(X, X_{0}, x_{0}\right) \rightarrow \check{\pi}_{k-1}^{*}\left(X_{0}, x_{0}\right)=\check{\pi}_{k-1}^{*}\left(X_{0},\left\{x_{0}\right\}, x_{0}\right)$ is introduced which induces a natural transformation. The corresponding sequence of the coarse shape groups is exact, although the shape sequence generally failed to be exact. This exactness makes powerful tool for computing coarse shape groups of some particular pointed pairs of metric compacta.
\end{abstract}

\section{INTRODUCTION}

The coarse shape theory was recently founded by the author and N. Uglešić ([2]). They have extended an abstract shape category by constructing an abstract coarse shape category. The (pointed) coarse shape category $S h^{*}\left(S h_{\star}^{*}\right)$, having (pointed) topological spaces as objects and having the (pointed) shape category $S h\left(S h_{\star}\right)$ as a subcategory is constructed. In the same way the pointed coarse shape category (of pairs) $S h_{\star}^{*}\left(S h_{\star}^{* 2}\right)$ having pointed topological spaces (pairs) for objects and having the pointed shape category (of pairs) $S h_{\star}\left(S h_{\star}^{2}\right)$ for its subcategory is constructed. The coarse

2010 Mathematics Subject Classification. 55P55, 55Q05, 55N99.

Key words and phrases. Polyhedron, inverse system, pro-category, pro*-category, expansion, shape, coarse shape, homotopy group, shape group, coarse shape group, exactness. 
shape theory functorially generalizes the shape theory such that there exist spaces (metrizable continua) having the same coarse shape type and different shape types (see $[1,2]$ ). However, the coarse shape preserves some important topological or shape invariants (see $[3,7]$ ) as connectedness, movability, strong movability, $n$-movability, shape dimension, triviality of shape, stability. There are also several important algebraic coarse shape invariants and, consequently, topological, homotopy and shape invariants. In [4], functors $\check{\pi}_{n}^{*}: S h_{\star}^{*} \rightarrow G r p$, $n \in \mathbb{N}$ (Grp denotes the category of groups) and $\check{\pi}_{0}^{*}: S h_{\star}^{*} \rightarrow S e t_{\star}$ are introduced. Here $S e t_{\star}$ denotes the category of pointed sets and base point preserving functions. Notice that we may consider every group as a pointed set having neutral element $o$ for a base point and every group homomorphism as a base point preserving function. In this paper we consider with more details functors $\check{\pi}_{n}^{*}: S h_{\star}^{* 2} \rightarrow \operatorname{Grp}, n>1, \check{\pi}_{1}^{*}: S h_{\star}^{* 2} \rightarrow S e t_{\star}$ which assigns to every pointed pair $\left(X, X_{0}, x_{0}\right)$ the $n$-th relative coarse shape group $\check{\pi}_{n}^{*}\left(X, X_{0}, x_{0}\right)$. Since every coarse shape group $\check{\pi}_{k}^{*}\left(X, x_{0}\right)$ can be considered as the relative coarse shape group $\check{\pi}_{k}^{*}\left(X,\left\{x_{0}\right\}, x_{0}\right)$, for $k \geqslant 2$, these groups are more general. Furthermore, every shape group $\check{\pi}_{n}\left(X, X_{0}, x_{0}\right)$ can be imbedded in $\check{\pi}_{n}^{*}\left(X, X_{0}, x_{0}\right)$ as its subgroup (Theorem 4.8). Therefore, the (relative) coarse shape groups provide better information on pointed (pairs of) spaces than the (relative) shape groups do. For every pointed pair of spaces $\left(X, X_{0}, x_{0}\right)$, where $X_{0}$ is normally embedded in $X$, and for every $k>1$, the boundary homomorphism $\partial_{k}^{*}: \check{\pi}_{k}^{*}\left(X, X_{0}, x_{0}\right) \rightarrow \check{\pi}_{k-1}^{*}\left(X_{0}, x_{0}\right)$ is introduced (Theorem 3.4). It induces natural transformation (Theorem 3.5). Further, it is natural to consider the sequence of the coarse shape groups of a pointed pair $\left(X, X_{0}, x_{0}\right)$. We know that the most useful feature of the relative homotopy groups $\pi_{k}\left(X, X_{0}, x_{0}\right)$ is that they fit into a long exact sequence. Further, the well known fact is that, even for a pointed pair $\left(X, X_{0}, x_{0}\right)$ of metric compacta, the sequence of the shape groups (the shape sequence) $\check{\pi}_{k}\left(X, X_{0}, x_{0}\right)$ generally failed to be exact. Nevertheless we proved that the sequence of the coarse shape groups of a pointed pair of metric compacta $\left(X, X_{0}, x_{0}\right)$ is exact (Corollary 4.3$)$. This makes powerful tool for calculating coarse shape groups of some particular pointed pairs of metric compacta as demonstrated by Examples 4.6 and 4.7. In Example 4.9 a pointed pair is given such that the corresponding shape sequence is not exact although that sequence is embedded in the exact sequence of the coarse shape groups.

\section{Preliminaries}

We recall some basic notions on the coarse shape category (see [2]). Let $X=\left(X_{\lambda}, p_{\lambda \lambda^{\prime}}, \Lambda\right)$ and $Y=\left(Y_{\mu}, q_{\mu \mu^{\prime}}, M\right)$ be two inverse systems in some category $\mathcal{C}$. An $S^{*}$-morphism of inverse systems, $\left(f, f_{\mu}^{n}\right): X \rightarrow Y$, consists of an index function $f: M \rightarrow \Lambda$, and of a set of $\mathcal{C}$-morphisms $f_{\mu}^{n}: X_{f(\mu)} \rightarrow Y_{\mu}$, $n \in \mathbb{N}, \mu \in M$, such that, for every related pair $\mu \leq \mu^{\prime}$ in $M$, there exists a 
$\lambda \in \Lambda, \lambda \geqslant f(\mu), f\left(\mu^{\prime}\right)$, and there exists an $n \in \mathbb{N}$ so that, for every $n^{\prime} \geqslant n$,

$$
f_{\mu}^{n^{\prime}} p_{f(\mu) \lambda}=q_{\mu \mu^{\prime}} f_{\mu^{\prime}}^{n^{\prime}} p_{f\left(\mu^{\prime}\right) \lambda} .
$$

If $M=\Lambda$ and the index function is the identity $1_{\Lambda}$ and, for every pair $\lambda \leq \lambda^{\prime}$, there exists an $n \in \mathbb{N}$ such that, for every $n^{\prime} \geq n, f_{\lambda}^{n^{\prime}} p_{\lambda \lambda^{\prime}}=q_{\lambda \lambda^{\prime}} f_{\lambda^{\prime}}^{n^{\prime}}$, then the $S^{*}$-morphism $\left(1_{\Lambda}, f_{\lambda}^{n}\right)$ is said to be level.

An $S^{*}$-morphism $\left(f, f_{\mu}^{n}\right): X \rightarrow Y$ of inverse systems in $\mathcal{C}$ is said to be equivalent to an $S^{*}$-morphism $\left(f^{\prime}, f_{\mu}^{\prime n}\right): X \rightarrow Y$, denoted by $\left(f, f_{\mu}^{n}\right) \sim$ $\left(f^{\prime}, f_{\mu}^{\prime n}\right)$, provided every $\mu \in M$ admits a $\lambda \in \Lambda, \lambda \geqslant f(\mu), f^{\prime}(\mu)$, and an $n \in \mathbb{N}$, such that, for every $n^{\prime} \geqslant n$,

$$
f_{\mu}^{n^{\prime}} p_{f(\mu) \lambda}=f_{\mu}^{\prime n^{\prime}} p_{f^{\prime}(\mu) \lambda} .
$$

The relation $\sim$ is an equivalence relation among $S^{*}$-morphisms of inverse systems in $\mathcal{C}$. The equivalence class $\left[\left(f, f_{\mu}^{n}\right)\right]$ of an $S^{*}$-morphism $\left(f, f_{\mu}^{n}\right): X \rightarrow$ $Y$ is denoted by $f^{*}$.

The category pro $^{*}-\mathcal{C}$ has as objects all inverse systems $X$ in $\mathcal{C}$ and as morphisms all equivalence classes $f^{*}=\left[\left(f, f_{\mu}^{n}\right)\right]$ of $S^{*}$-morphisms $\left(f, f_{\mu}^{n}\right)$. The composition in pro*-C is well defined by putting $g^{*} f^{*}=h^{*} \equiv\left[\left(h, h_{\nu}^{n}\right)\right]$, where $\left(h, h_{\nu}^{n}\right)=\left(g, g_{\nu}^{n}\right)\left(f, f_{\mu}^{n}\right)=\left(f g, g_{\nu}^{n} f_{g(\nu)}^{n}\right)$. For every inverse system $X$ in $\mathcal{C}$, the identity morphism in pro ${ }^{*} \mathcal{C}$ is $1_{X}^{*}=\left[\left(1_{\Lambda}, 1_{X_{\lambda}}\right)\right]$, where $1_{\Lambda}$ is the identity function and $1_{X_{\lambda}}^{n}=1_{X_{\lambda}}$ are the identity morphisms in $\mathcal{C}$, for all $n \in \mathbb{N}$ and $\lambda \in \Lambda$.

A functor $\underline{J} \equiv \underline{J}_{\mathcal{C}}:$ pro-C $\rightarrow$ pro*-C keeps objects fixed, i.e., $\underline{J}(X)=X$, for every inverse system $X$ in $\mathcal{C}$, and to each morphism $f=\left[\left(f, f_{\mu}\right)\right] \in$ pro$\mathcal{C}(X, Y)$ it assigns a morphism $\underline{J}(f)=f^{*}=\left[\left(f, f_{\mu}^{n}\right)\right] \in \operatorname{pro}^{*}-\mathcal{C}(X, Y)$, which is said to be induced by $f$, where $f_{\mu}^{n}=f_{\mu}$ for all $\mu \in M$ and $n \in \mathbb{N}$. Since the functor $\underline{J}$ is faithful, we may consider the category pro-C as a subcategory of pro $^{*}-\mathcal{C}$.

Let us consider any category pair $(\mathcal{C}, \mathcal{D})$ where $\mathcal{D}$ is a full and pro-reflective (i.e., dense) subcategory of $\mathcal{C}$ (see [5, I.2.2]). Let $p: X \rightarrow X$ and $p^{\prime}: X \rightarrow X^{\prime}$ be $\mathcal{D}$-expansions of the same object $X$ of $\mathcal{C}$, and let $q: Y \rightarrow Y$ and $q^{\prime}: Y \rightarrow Y^{\prime}$ be $\mathcal{D}$-expansions of the same object $Y$ of $\mathcal{C}$ (see [5, I.2.1]). Therefore there exist two unique isomorphisms $i: X \rightarrow X^{\prime}$ and $j: Y \rightarrow Y^{\prime}$ in pro-D. Consequently, $i^{*} \equiv \underline{J}(i): X \rightarrow X^{\prime}$ and $j^{*} \equiv \underline{J}(j): Y \rightarrow Y^{\prime}$ are isomorphisms in pro*-D. A morphism $f^{*}: X \rightarrow Y$ is said to be pro*-D equivalent to a morphism $f^{\prime *}: X^{\prime} \rightarrow Y^{\prime}$, denoted by $f^{*} \sim f^{\prime *}$, provided the following diagram in pro $^{*}-\mathcal{D}$ commutes:

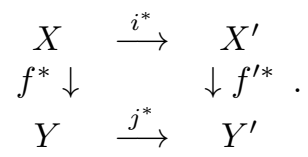

Hereby is defined an equivalence relation on the appropriate subclass of morphisms of rro $^{*}-\mathcal{D}$. The equivalence class of $f^{*}$ is denoted by $\left\langle f^{*}\right\rangle$. 
We define the (abstract) coarse shape category $S h_{(\mathcal{C}, \mathcal{D})}^{*}$ for $(\mathcal{C}, \mathcal{D})$ as the category whose objects are all the objects of $\mathcal{C}$ and whose morphisms $F^{*} \in$ $S h_{(\mathcal{C}, \mathcal{D})}^{*}(X, Y)$ are all pro*-D equivalence class $\left\langle f^{*}\right\rangle$ of morphisms $f^{*}: X \rightarrow Y$, with respect to any choice of a pair of $\mathcal{D}$-expansions $p: X \rightarrow X, q: Y \rightarrow$ $Y$. The composition of an $F^{*}: X \rightarrow Y, F^{*}=\left\langle f^{*}\right\rangle$ and a $G^{*}: Y \rightarrow Z$, $G^{*}=\left\langle g^{*}\right\rangle$, is defined by the representatives, i.e., $G^{*} F^{*}: X \rightarrow Z, G^{*} F^{*}=$ $\left\langle g^{*} f^{*}\right\rangle$. The identity coarse shape morphism on an object $X, 1_{X}^{*}: X \rightarrow X$, is the pro $^{*}-\mathcal{D}$ equivalence class $\left\langle 1_{X}^{*}\right\rangle$ of the identity morphism $1_{X}^{*}$ in pro $^{*}-\mathcal{D}$. Since $S h_{(\mathcal{C}, \mathcal{D})}^{*}(X, Y) \approx$ pro* $^{*} \mathcal{D}(X, Y)$, one may say that pro*-D is the realizing category for the coarse shape category $S h_{(\mathcal{C}, \mathcal{D})}^{*}$ in the same way as pro- $\mathcal{D}$ is for the shape category $S h_{(\mathcal{C}, \mathcal{D})}$. The functor $\underline{J}$ of the "pro-categories" induces the embedding functor $J \equiv J_{(\mathcal{C}, \mathcal{D})}: S h_{(\mathcal{C}, \mathcal{D})} \rightarrow S h_{(\mathcal{C}, \mathcal{D})}^{*}$ of the "shape" categories in such a manner that $J$ keeps the objects fixed and to each shape morphism $F=\langle f\rangle \in S h_{(\mathcal{C}, \mathcal{D})}(X, Y)$ represented by a morphism $f$ of pro- $\mathcal{D}$ category it assigns the coarse shape morphism $F^{*}=\left\langle f^{*}\right\rangle=J(F) \in S h_{(\mathcal{C}, \mathcal{D})}^{*}(X, Y)$ which is represented by the morphism $f^{*}=\underline{J}(f)$ of pro $^{*}-\mathcal{D}$ induced by $f$.

In this paper $\mathcal{C}$ will be the pointed homotopy category HTop $p_{\star}$ or the pointed homotopy category of pairs $H T o p_{\star}^{2}$. Recall that objects of the category $H T o p_{\star}^{2}$ are all the pointed pairs of topological spaces, $\left(X, X_{0}, x_{0}\right)$, $x_{0} \in X_{0} \subseteq X$ and morphisms are all the homotopy classes $[f]$ of mappings of pointed pairs, $f:\left(X, X_{0}, x_{0}\right) \rightarrow\left(Y, Y_{0}, y_{0}\right)$, i.e., mappings $f: X \rightarrow Y$ satisfying $f\left(X_{0}\right) \subseteq Y_{0}$ and $f\left(x_{0}\right)=y_{0}$, (it is understood that a homotopy passes through maps of the same form). Objects of the category HTop $p_{\star}$ are all the pointed spaces $\left(X, x_{0}\right)$ and morphisms are all the homotopy classes $[f]$ of mappings of pointed spaces, $f:\left(X, x_{0}\right) \rightarrow\left(Y, y_{0}\right)$. In this paper the homotopy class $[f]$ of a map $f$ (briefly $H$-map), i.e., a morphism of the category HTop $p_{\star}$ or $H T o p_{\star}^{2}$, will be usually denoted by omitting the brackets, unless we already have used this notation for some continuous mapping. A reduction in the object classes to all pointed polyhedral pairs and pointed polyhedra yields the full subcategories $H P o l_{\star}^{2} \subseteq H T o p_{\star}^{2}$ and $H P o l_{\star} \subseteq H T o p_{\star}$ respectively. The well known fact is that $H P o l_{\star}^{2}$, and $H \mathrm{Pol}_{\star}$ are pro-reflective subcategories of $H T o p_{\star}^{2}$ and $H T o p_{\star}$ respectively ([5, Theorem I.4.7, Theorem I.4.8]). This means that, for every pointed pair of topological spaces $\left(X, X_{0}, x_{0}\right)$, there exists a $H$ Pol $_{\star}^{2}$-expansion of $\left(X, X_{0}, x_{0}\right)$, i.e., an inverse system $\left(X, X_{0}, x_{0}\right)=\left(\left(X_{\lambda}, X_{0 \lambda}, x_{0 \lambda}\right), p_{\lambda \lambda^{\prime}}, \Lambda\right)$ in $\mathrm{HPol}_{\star}^{2}$ and a morphism $p=\left[\left(p_{\lambda}\right)\right]:\left(X, X_{0}, x_{0}\right) \rightarrow\left(X, X_{0}, x_{0}\right)$ of pro$H T o p_{\star}^{2}$ satisfying some special properties (see [5, Theorem I.2.1]). Similarly, for every pointed space $\left(X, x_{0}\right)$ there exists a $H P l_{\star}$-expansion of $\left(X, x_{0}\right)$, i.e., an inverse system $\left(X, x_{0}\right)=\left(\left(X_{\lambda}, x_{0 \lambda}\right), p_{\lambda \lambda^{\prime}}, \Lambda\right)$ in $H P o l_{\star}$ and a morphism $p=\left[\left(p_{\lambda}\right)\right]:\left(X, x_{0}\right) \rightarrow\left(X, x_{0}\right)$ of $p r o-H T o p_{\star}$ satisfying some special properties (see [5, Theorem I.2.1]). 
The construction of the pointed coarse shape category of pairs $S h_{\star}^{* 2}$ follows now the general rule, i.e., it is the category $S h_{\left(H T o p_{\star}^{2}, H P o l_{\star}^{2}\right)}^{*}$. Briefly, the objects of $S h_{\star}^{* 2}$ are all pointed pairs of topological spaces $\left(X, X_{0}, x_{0}\right)$, while a morphism set $S h_{\star}^{* 2}\left(\left(X, X_{0}, x_{0}\right),\left(Y, Y_{0}, y_{0}\right)\right)$ consists of all the equivalence classes $F^{*}=\left\langle f^{*}\right\rangle$ of morphisms $f^{*}=\left[\left(f, f_{\mu}^{n}\right)\right]:\left(X, X_{0}, x_{0}\right) \rightarrow\left(Y, Y_{0}, y_{0}\right)$ of pro* $^{*}-\mathrm{HPol}_{\star}^{2}$ ranging all over the corresponding expansions. A morphism $f^{*}$ is represented by an $S^{*}$-morphism $\left(f, f_{\mu}^{n}\right):\left(\left(X_{\lambda}, X_{0 \lambda}, x_{0 \lambda}\right), p_{\lambda \lambda^{\prime}}, \Lambda\right) \rightarrow$ $\left(\left(Y_{\mu}, Y_{0 \mu}, y_{0 \mu}\right), q_{\mu \mu^{\prime}}, M\right)$, where $f_{\mu}^{n}:\left(X_{f(\mu)}, X_{0 f(\mu)}, x_{0 f(\mu)}\right) \rightarrow\left(Y_{\mu}, Y_{0 \mu}, y_{0 \mu}\right)$ is a $H$-map, i.e., a morphism of $H_{P o l}^{2}$, for every $\mu \in M, n \in \mathbb{N}$. Therefore, the category pro $^{*}-H P o l_{\star}^{2}$ is the realizing category for $S h_{\star}^{* 2}$, i.e.,

$$
S h_{\star}^{* 2}\left(\left(X, X_{0}, x_{0}\right),\left(Y, Y_{0}, y_{0}\right)\right) \approx \operatorname{pro}^{*}-H \operatorname{Pol}_{\star}^{2}\left(\left(X, X_{0}, x_{0}\right),\left(Y, Y_{0}, y_{0}\right)\right),
$$

and every coarse shape morphism $F^{*}:\left(X, X_{0}, x_{0}\right) \rightarrow\left(Y, Y_{0}, y_{0}\right)$ is represented by a diagram in pro $^{*}-H T o p_{\star}^{2}$

$$
\begin{aligned}
& \left(X, X_{0}, x_{0}\right) \stackrel{p}{\leftarrow}\left(X, X_{0}, x_{0}\right) \\
& f^{*} \downarrow \\
& \left(Y, Y_{0}, y_{0}\right) \underset{q}{\leftarrow}\left(Y, Y_{0}, y_{0}\right)
\end{aligned}
$$

Similarly, one constructs the pointed coarse shape category

$$
S h_{\star}^{*}=S h_{\left(H T o p_{\star}, H P o l_{\star}\right)}^{*}
$$

of pointed topological spaces having the category pro $^{*}-\mathrm{HPol}_{\star}$ for its realizing category. The embedding functor $J: S h_{\star}^{2} \rightarrow S h_{\star}^{* 2}\left(J: S h_{\star} \rightarrow S h_{\star}^{*}\right)$ relates the pointed shape category (of pairs) with the corresponding coarse shape category.

For a pointed topological pair $\left(X, X_{0}, x_{0}\right)$, where the subspace $X_{0}$ is normally embedded in $X$ (see [5, II. 3.3,I. 6.5]), there exists an $\mathrm{HPol}_{\star}^{2}$ expansion

$$
p=\left(p_{\lambda}\right):\left(X, X_{0}, x_{0}\right) \rightarrow\left(X, X_{0}, x_{0}\right)=\left(\left(X_{\lambda}, X_{0 \lambda}, x_{0 \lambda}\right), p_{\lambda \lambda^{\prime}}, \Lambda\right)
$$

such that $p:\left(X, x_{0}\right) \rightarrow\left(X, x_{0}\right)$ and

$$
\left.p\right|_{X_{0}}=\left(\left.p_{\lambda}\right|_{X_{0}}\right):\left(X_{0}, x_{0}\right) \rightarrow\left(X_{0}, x_{0}\right)=\left(\left(X_{0 \lambda}, x_{0 \lambda}\right),\left.p_{\lambda \lambda^{\prime}}\right|_{X_{0 \lambda^{\prime}}}, \Lambda\right)
$$

are $H \mathrm{Pol}_{\star}$-expansions. We will say that this expansion is a normal $\mathrm{HPol}_{\star^{-}}^{2}$ expansion (of a pointed pair). Therefore, if $X_{0}$ and $Y_{0}$ are normally embedded in $X$ and $Y$, respectively, then for every coarse shape morphism $F^{*}:\left(X, X_{0}, x_{0}\right) \rightarrow\left(Y, Y_{0}, y_{0}\right)$ in $S h_{\star}^{* 2}$ which is represented by $f^{*}=\left[\left(f, f_{\mu}^{n}\right)\right]:$ $\left(X, X_{0}, x_{0}\right) \rightarrow\left(Y, Y_{0}, y_{0}\right)$, there exists the restricted coarse shape morphism $\left.F^{*}\right|_{\left(X_{0}, x_{0}\right)}:\left(X_{0}, x_{0}\right) \rightarrow\left(Y_{0}, y_{0}\right)$ in $S h_{\star}^{*}$ which is defined via the representative $\left.f^{*}\right|_{\left(X_{0}, x_{0}\right)}=\left[\left(f,\left.f_{\mu}^{n}\right|_{X_{0 f(\mu)}}\right)\right]:\left(X_{0}, x_{0}\right) \rightarrow\left(Y_{0}, y_{0}\right)$, where $p:\left(X, X_{0}, x_{0}\right) \rightarrow$ $\left(X, X_{0}, x_{0}\right)$ and $q:\left(Y, Y_{0}, y_{0}\right) \rightarrow\left(Y, Y_{0}, y_{0}\right)$ are normal $H P o l_{\star}^{2}$-expansions. 
THEOREM 2.1. Every pointed pair of metric compacta $\left(X, X_{0}, x_{0}\right)$ is the limit of a "countable" inverse system of pointed compact polyhedral pairs and continuous bonding maps, i.e., there exists an inverse limit $\left(p_{\lambda}\right)$ : $\left(X, X_{0}, x_{0}\right) \rightarrow\left(\left(X_{\lambda}, X_{0 \lambda}, x_{0 \lambda}\right), p_{\lambda \lambda^{\prime}}, \Lambda\right)$ satisfying card $\Lambda=\aleph_{0}$. Further, $p=\left(\left[p_{\lambda}\right]\right):\left(X, X_{0}, x_{0}\right) \rightarrow\left(\left(X_{\lambda}, X_{0 \lambda}, x_{0 \lambda}\right),\left[p_{\lambda \lambda^{\prime}}\right], \Lambda\right)$ is the normal HPol ${ }_{\star}^{2}-$ expansion of $\left(X, X_{0}, x_{0}\right)$.

Proof. According to [5, Theorems I.5.11. and I.5.10.] there exists an inverse system $\left(\left(X_{\lambda}, X_{0 \lambda}\right), p_{\lambda \lambda^{\prime}}, \Lambda\right)$ of pairs of compact polyhedra satisfying $\operatorname{card} \Lambda=\aleph_{0}$, and there exists a morphism $\left(p_{\lambda}\right):\left(X, X_{0}\right) \rightarrow\left(\left(X_{\lambda}, X_{0 \lambda}\right), p_{\lambda \lambda^{\prime}}, \Lambda\right)$ of pro- $c \mathcal{M}^{2}(c \mathcal{M}$ denotes the category having metric compacta for the objects and having continuous mappings as the morphisms) which is the inverse limit (in the category of pairs of metric compacta $c \mathcal{M}^{2}$ ) such that $\left(p_{\lambda}\right): X \rightarrow\left(X_{\lambda}, p_{\lambda \lambda^{\prime}}, \Lambda\right)$ and $\left(\left.p_{\lambda}\right|_{X_{0}}\right): X_{0} \rightarrow\left(X_{0 \lambda},\left.p_{\lambda \lambda^{\prime}}\right|_{X_{0 \lambda^{\prime}}}, \Lambda\right)$ are the inverse limits (in the category $c \mathcal{M}$ ). One can easily prove that

$$
\begin{aligned}
& \left(p_{\lambda}\right):\left(X, X_{0}, x_{0}\right) \rightarrow\left(\left(X_{\lambda}, X_{0 \lambda}, x_{0 \lambda}\right), p_{\lambda \lambda^{\prime}}, \Lambda\right), \\
& \left(p_{\lambda}\right):\left(X, x_{0}\right) \rightarrow\left(\left(X_{\lambda}, x_{0 \lambda}\right), p_{\lambda \lambda^{\prime}}, \Lambda\right)
\end{aligned}
$$

and

$$
\left(\left.p_{\lambda}\right|_{X_{0}}\right): X_{0} \rightarrow\left(\left(X_{0 \lambda}, x_{0 \lambda}\right),\left.p_{\lambda \lambda^{\prime}}\right|_{X_{0 \lambda^{\prime}}}, \Lambda\right), p_{\lambda}\left(x_{0}\right)=x_{0 \lambda}, \lambda \in \Lambda,
$$

are the inverse limits (in the category of the pointed pairs of metric compacta $c \mathcal{M}_{\star}^{2}$ and the category of the pointed metric compacta $c \mathcal{M}_{\star}$ respectively). Now, in order to prove the second assertion, one can argue as in the proof of $[5$, Theorem I.5.13.] (nonpointed case). Namely, that proof allows straightforward translation to the pointed case.

\section{THE BOUNDARY HOMOMORPHISM OF THE COARSE SHAPE GROUPS}

We begin by recalling some notions concerning the (relative) homotopy groups and the (relative) coarse shape groups introduced in [4].

Recall that, for every pointed pair of topological space $\left(X, X_{0}, x_{0}\right)$ and for every $k \in \mathbb{N}$, elements of the relative $k$-dimensional homotopy group $\pi_{k}\left(X, X_{0}, x_{0}\right)$ can be regarded as homotopy classes of maps $\left(D^{k}, S^{k-1}, s_{0}\right) \rightarrow$ $\left(X, X_{0}, x_{0}\right)$, where $D^{k}$ denotes the standard $k$-dimensional disk having the $(k-1)$-dimensional sphere $S^{k-1}$ for its boundary. A group operation is defined in $\pi_{k}\left(X, X_{0}, x_{0}\right)$ for $k \geqslant 2$ and we will use the additive notation for it. The neutral element of $\pi_{k}\left(X, X_{0}, x_{0}\right)$, i.e., the homotopy class of the constant map to $x_{0}$, we will denote by $o:\left(D^{k}, S^{k-1}, s_{0}\right) \rightarrow\left(X, X_{0}, x_{0}\right)$. The trivial homotopy group $\pi_{k}\left(X, X_{0}, x_{0}\right)=\{o\}$ we will denote by 0 , same as any other trivial group. For every $k \in \mathbb{N} \backslash\{1\}$ and for every pointed space $\left(X, x_{0}\right)$, the $k$-th homotopy group $\pi_{k}\left(X, x_{0}\right)$ is defined as $\pi_{k}\left(X,\left\{x_{0}\right\}, x_{0}\right)$. Since we may identify every map $\left(D^{k}, S^{k-1}, s_{0}\right) \rightarrow\left(X,\left\{x_{0}\right\}, x_{0}\right)$ with a map of the quotient $D^{k} / S^{k-1}=S^{k}$ to $X$ (the base point $s_{0}=S^{k-1} / S^{k-1}$ to $x_{0}$ ), we can also view 
elements of $\pi_{k}\left(X, x_{0}\right)$ as homotopy classes of maps $\left(S^{k}, s_{0}\right) \rightarrow\left(X, x_{0}\right)$. Using this approach to homotopy groups, the definition of $\pi_{k}\left(X, x_{0}\right)$ is extended to all $k \in \mathbb{N}_{0}$ and a group operation is defined in $\pi_{1}\left(X, x_{0}\right)$ as well.

For every $k \in \mathbb{N}$ and every pointed pair of spaces $\left(X, X_{0}, x_{x_{0}}\right)$, the $k$-th relative coarse shape group of a pair $\left(X, X_{0}, x_{0}\right)$ at a base point $x_{0}$, denoted by $\check{\pi}_{k}^{*}\left(X, X_{0}, x_{0}\right)$, is defined. Its underlying set consists of all coarse shape morphisms $A^{*}:\left(D^{k}, S^{k-1}, s_{0}\right) \rightarrow\left(X, X_{0}, x_{0}\right)$ in the category $S h_{\star}^{* 2}$. For every $k \geqslant 2$, a group operation + is given by formula

$$
A^{*}+B^{*}=\left\langle a^{*}\right\rangle+\left\langle b^{*}\right\rangle=\left\langle a^{*}+b^{*}\right\rangle=\left\langle\left[\left(a_{\lambda}^{n}\right)\right]+\left[\left(b_{\lambda}^{n}\right)\right]\right\rangle=\left\langle\left[\left(a_{\lambda}^{n}+b_{\lambda}^{n}\right)\right]\right\rangle
$$

where coarse shape morphisms $A^{*}$ and $B^{*}$ are represented by morphisms $a^{*}=\left[\left(a_{\lambda}^{n}\right)\right]$ and $b^{*}=\left[\left(b_{\lambda}^{n}\right)\right]:\left(D^{k}, S^{k-1}, s_{0}\right) \rightarrow\left(X, X_{0}, x_{0}\right)$ in pro*-HPol ${ }_{\star}^{2}$, respectively, and

$$
p:\left(X, X_{0}, x_{0}\right) \rightarrow\left(X, X_{0}, x_{0}\right)=\left(\left(X_{\lambda}, X_{0 \lambda}, x_{0 \lambda}\right), p_{\lambda \lambda^{\prime}}, \Lambda\right)
$$

is an $\mathrm{HPol}_{\star}^{2}$-expansion of a pointed pair $\left(X, X_{0}, x_{0}\right)$. Notice that the sum $a_{\lambda}^{n}+$ $b_{\lambda}^{n}$ in (3.1) denotes the $H$-map which is the sum in the group $\pi_{k}\left(X_{\lambda}, X_{0 \lambda}, x_{0 \lambda}\right)$. For every $k \geq 2, \check{\pi}_{k}^{*}\left(X, X_{0}, x_{0}\right)$ is a group (for $k \geq 3$ an abelian group) and $\check{\pi}_{1}^{*}\left(X, X_{0}, x_{0}\right)$ is a pointed set. The neutral element (base point) in $\check{\pi}_{k}^{*}\left(X, X_{0}, x_{0}\right)$ is a coarse shape morphism $O^{*}=\left\langle o^{*}\right\rangle, o^{*}=\left[\left(o_{\lambda}^{n}\right)\right]:\left(D^{k}, S^{k-1}\right.$, $\left.s_{0}\right) \rightarrow\left(X, X_{0}, x_{0}\right)$, where $o_{\lambda}^{n}:\left(D^{k}, S^{k-1}, s_{0}\right) \rightarrow\left(X_{\lambda}, X_{0 \lambda}, x_{0 \lambda}\right)$ denotes the neutral element of $\pi_{k}\left(X_{\lambda}, X_{0 \lambda}, x_{0 \lambda}\right), \lambda \in \Lambda$.

For every $k \in \mathbb{N}_{0}$ and every pointed space $\left(X, x_{0}\right)$ the $k$-th coarse shape group $\check{\pi}_{k}^{*}\left(X, x_{0}\right)$ of a pointed space $\left(X, x_{0}\right)$ at a base point $x_{0}$ is defined. For $k=0$ it is the pointed set $S h_{\star}^{*}\left(\left(S^{0}, s_{0}\right),\left(X, x_{0}\right)\right)$ and for every $k \geqslant 1$ it is a group whose underlaying set is $S h_{\star}^{*}\left(\left(S^{k}, s_{0}\right),\left(X, x_{0}\right)\right)$ and a group operation + is given by formula (3.1), where coarse shape morphisms $A^{*}$ and $B^{*}$ are represented by morphisms $a^{*}=\left[\left(a_{\lambda}^{n}\right)\right]$ and $b^{*}=\left[\left(b_{\lambda}^{n}\right)\right]:\left(S^{k}, s_{0}\right) \rightarrow\left(X, x_{0}\right)$ in pro*$^{*}-\mathrm{HPol}_{\star}$, respectively, and

$$
p:\left(X, x_{0}\right) \rightarrow\left(X, x_{0}\right)=\left(\left(X_{\lambda}, x_{0 \lambda}\right), p_{\lambda \lambda^{\prime}}, \Lambda\right)
$$

is an $H P o l_{\star}$-expansion of a pointed space $\left(X, x_{0}\right)$. Hereby the sum $a_{\lambda}^{n}+b_{\lambda}^{n}$ in (3.1) denotes the $H$-map which is the sum in the group $\pi_{k}\left(X_{\lambda}, x_{0 \lambda}\right)$. Since, an $H P o l_{\star}^{2}$-expansion of a pointed pair $\left(X,\left\{x_{0}\right\}, x_{0}\right)$ is

$$
p:\left(X,\left\{x_{0}\right\}, x_{0}\right) \rightarrow\left(X, x_{0}, x_{0}\right)=\left(\left(X_{\lambda},\left\{x_{0 \lambda}\right\}, x_{0 \lambda}\right), p_{\lambda \lambda^{\prime}}, \Lambda\right),
$$

and since every $H$-map $\left(D^{k}, S^{k-1}, s_{0}\right) \rightarrow\left(X,\left\{x_{0}\right\}, x_{0}\right)$ can be regarded as an $H$-map $\left(S^{k}, s_{0}\right) \rightarrow\left(X, x_{0}\right)$, we may identify the set $S h_{\star}^{*}\left(\left(S^{k}, s_{0}\right),\left(X, x_{0}\right)\right)$ with the set $S h_{\star}^{* 2}\left(\left(D^{k}, S^{k-1}, s_{0}\right),\left(X,\left\{x_{0}\right\}, x_{0}\right)\right)$, for every $k \in \mathbb{N}$. Therefore, $\pi_{k}\left(X, x_{0}\right)=\pi_{k}\left(X,\left\{x_{0}\right\}, x_{0}\right)$ implies that one may consider the coarse shape group $\check{\pi}_{k}^{*}\left(X, x_{0}\right)$ as the relative coarse shape group $\check{\pi}_{k}^{*}\left(X,\left\{x_{0}\right\}, x_{0}\right)$, for every $k \geqslant 2$. 
In [4, Example 1], an explicit formula for the coarse shape group

$$
\check{\pi}_{k}^{*}\left(P, p_{0}\right)=\prod_{n \in \mathbb{N}} \pi_{k}\left(P, p_{0}\right) / \bigoplus_{n \in \mathbb{N}} \pi_{k}\left(P, p_{0}\right)
$$

of a pointed polyhedron $\left(P, p_{0}\right)$, for every $k \in \mathbb{N}$, is given. A proof similar to the one given in $[4]$ gives rise to the following generalization of that formula:

Proposition 3.1. Given a pointed polyhedral pair $\left(P, P_{0}, p_{0}\right)$ and a $k \in$ $\mathbb{N} \backslash\{1\}$, the coarse shape group $\check{\pi}_{k}^{*}\left(P, P_{0}, p_{0}\right)$ is the quotient group

$$
\left(\prod_{n \in \mathbb{N}} G_{n}\right) /\left(\bigoplus_{n \in \mathbb{N}} G_{n}\right),
$$

where $G_{n}=\pi_{k}\left(P, P_{0}, p_{0}\right), n \in \mathbb{N}$.

We may also establish the corresponding formula for the coarse shape group $\check{\pi}_{0}^{*}\left(P, p_{0}\right)$ as well as for the $\check{\pi}_{1}^{*}\left(P, P_{0}, p_{0}\right)$, where a group structure is not defined. Namely, it holds that $\check{\pi}_{0}^{*}\left(P, p_{0}\right)=\prod_{n \in \mathbb{N}} \pi_{0}\left(P, p_{0}\right) /(\sim)$ and $\check{\pi}_{1}^{*}\left(P, P_{0}, p_{0}\right)=\prod_{n \in \mathbb{N}} \pi_{1}\left(P, P_{0}, p_{0}\right) /(\sim)$, where $\sim$ denotes an equivalence relation on the direct product of pointed sets $\prod_{n \in \mathbb{N}} G_{n}$ given by the rule: $\left(g_{1}, g_{2}, \ldots\right) \sim\left(g_{1}^{\prime}, g_{2}^{\prime}, \ldots\right)$ provided there exists an $n_{0} \in \mathbb{N}$ such that $g_{n}=g_{n}^{\prime}$, for every $n \geqslant n_{0}$.

For every $k \in \mathbb{N}$ and for every coarse shape morphism $F^{*}:\left(X, X_{0}, x_{0}\right) \rightarrow$ $\left(Y, Y_{0}, y_{0}\right)$ of $S h_{\star}^{* 2}$, a homomorphism (a base point preserving function, for $k=1)$

$$
\check{\pi}_{k}^{*}\left(F^{*}\right): \check{\pi}_{k}^{*}\left(X, X_{0}, x_{0}\right) \rightarrow \check{\pi}_{k}^{*}\left(Y, Y_{0}, y_{0}\right)
$$

is defined by the following rule

$$
\check{\pi}_{k}^{*}\left(F^{*}\right)\left(A^{*}\right)=F^{*} A^{*},
$$

for every $A^{*} \in \check{\pi}_{k}^{*}\left(X, X_{0}, x_{0}\right)$. By following this rule, for every $k \in \mathbb{N}_{0}$, a homomorphism (a base point preserving function) $\check{\pi}_{k}^{*}\left(F^{*}\right): \check{\pi}_{k}^{*}\left(X, x_{0}\right) \rightarrow$ $\check{\pi}_{k}^{*}\left(Y, y_{0}\right)$ is defined for every coarse shape morphism $F^{*}:\left(X, x_{0}\right) \rightarrow\left(Y, y_{0}\right)$ of $S h_{\star}^{*}$.

Recall that, for every $k \in \mathbb{N}$, and for every pointed topological pair $\left(X, X_{0}, x_{0}\right)$, there exists a homotopy boundary homomorphism (for $k=1$ a base point preserving function) $\partial_{k} \equiv \partial_{k}\left(X, X_{0}, x_{0}\right): \pi_{k}\left(X, X_{0}, x_{0}\right) \rightarrow$ $\pi_{k-1}\left(X_{0}, x_{0}\right)$ defined by the restriction of mappings:

$$
\partial_{k}(a)=a \mid\left(S^{k-1}, s_{0}\right):\left(S^{k-1}, s_{0}\right) \rightarrow\left(X_{0}, x_{0}\right),
$$

for all $H$-maps $a:\left(D^{k}, S^{k-1}, s_{0}\right) \rightarrow\left(X, X_{0}, x\right)$. 
Further, letting $i:\left(X_{0}, x_{0}\right) \rightarrow\left(X, x_{0}\right)$ and $j:\left(X,\left\{x_{0}\right\}, x_{0}\right) \rightarrow\left(X, X_{0}, x_{0}\right)$ to be the homotopy classes of inclusions, the following sequence

$$
\begin{aligned}
& \ldots \stackrel{\partial_{k+1}}{\rightarrow} \pi_{k}\left(X_{0}, x_{0}\right) \stackrel{\pi_{k}(i)}{\rightarrow} \pi_{k}\left(X, x_{0}\right) \stackrel{\pi_{k}(j)}{\rightarrow} \pi_{k}\left(X, X_{0}, x_{0}\right) \stackrel{\partial_{k}}{\longrightarrow} \ldots \\
& \ldots \stackrel{\partial_{f}}{\rightarrow} \pi_{0}\left(X_{0}, x_{0}\right) \stackrel{\pi_{0}(i)}{\rightarrow} \pi_{0}\left(X, x_{0}\right)
\end{aligned}
$$

is exact. Recall, that a sequence

$$
\cdots \rightarrow X^{\prime} \stackrel{f^{\prime}}{\rightarrow} X \stackrel{f}{\rightarrow} X^{\prime \prime} \rightarrow \cdots
$$

of group homomorphisms is said to be exact (semiexact) at term $X$ provided $\operatorname{Im} f^{\prime}=\operatorname{ker} f\left(\operatorname{Im} f^{\prime} \subseteq \operatorname{ker} f\right)$. Exactness and semiexactness at $X$ still make sense if some of terms $X^{\prime}, X$, and $X^{\prime \prime}$ are pointed sets having no group structures and $f$ is a base point preserving function. In that case we consider ker $f$ as the preimage $f^{-1}(o)$ of the base point (or neutral element) o. A sequence of homomorphisms is exact if it is exact at each of its terms. A sequence of homomorphisms is said to be a chain if it is semiexact at each of its terms.

A useful reformulation of what is meant by exactness of the sequence (3.3) at the term $\pi_{k}\left(X, x_{0}\right)$ is given as follows.

Proposition 3.2. Let $x_{0} \in X_{0} \subseteq X$ and let $a:\left(S^{k}, s_{0}\right) \rightarrow\left(X, x_{0}\right)$ be an $H$-map. The following assertions are equivalent:

(i) There exists an H-map $b:\left(S^{k}, s_{0}\right) \rightarrow\left(X_{0}, x_{0}\right)$ such that $i \circ b=a$

(ii) If we view a as an $H$-map $\alpha:\left(D^{k}, S^{k-1}, s_{0}\right) \rightarrow\left(X,\left\{x_{0}\right\}, x_{0}\right)$, then $j \circ \alpha:\left(D^{k}, S^{k-1}, s_{0}\right) \rightarrow\left(X, X_{0}, x_{0}\right)$ coincides witho $:\left(D^{k}, S^{k-1}, s_{0}\right) \rightarrow$ $\left(X, X_{0}, x_{0}\right)$.

REMARK 3.3. One can easily establish an analogue characterization of exactness of the sequence (3.3) at any other term.

For every pointed topological pair $\left(X, X_{0}, x_{0}\right)$, where $X_{0}$ is normally embedded in $X$, and every $k \in \mathbb{N}$, we are now able to define the boundary homomorphism of the coarse shape groups

$$
\partial_{k}^{*}: \check{\pi}_{k}^{*}\left(X, X_{0}, x_{0}\right) \rightarrow \check{\pi}_{k-1}^{*}\left(X_{0}, x_{0}\right)
$$

given by restricting coarse shape morphisms $A^{*}:\left(D^{k}, S^{k-1}, s_{0}\right) \rightarrow\left(X, X_{0}, x_{0}\right)$ to $\left(S^{k-1}, s_{0}\right)$, i.e.,

$$
\partial_{k}^{*}\left(A^{*}\right)=\left.A^{*}\right|_{\left(S^{k-1}, s_{0}\right)}:\left(S^{k-1}, s_{0}\right) \rightarrow\left(X_{0}, x_{0}\right),
$$

for every $A^{*} \in \check{\pi}_{k}^{*}\left(X, X_{0}, x_{0}\right)$. Notice that if $A^{*}$ is represented by a morphism $a^{*}=\left[\left(a_{\lambda}^{n}\right)\right]:\left(D^{k}, S^{k-1}, s_{0}\right) \rightarrow\left(X, X_{0}, x_{0}\right)$ of $\operatorname{pro}^{*}-H P o l_{\star}^{2}$, then $\partial_{k}^{*}\left(A^{*}\right)$ is represented by the morphism $\left.a^{*}\right|_{\left(S^{k-1}, s_{0}\right)}=\left[\left(\left.a_{\lambda}^{n}\right|_{\left(S^{k-1}, s_{0}\right)}\right)\right]:\left(S^{k-1}, s_{0}\right) \rightarrow$ $\left(X_{0}, x_{0}\right)$ of pro $^{*}-H P l_{\star}$, where

$$
p:\left(X, X_{0}, x_{0}\right) \rightarrow\left(X, X_{0}, x_{0}\right)=\left(\left(X_{\lambda}, X_{0 \lambda}, x_{0 \lambda}\right), p_{\lambda \lambda^{\prime}}, \Lambda\right)
$$


is a normal $H P o l_{\star}^{2}$-expansion of a pointed pair $\left(X, X_{0}, x_{0}\right)$. Since, $\left.a_{\lambda}^{n}\right|_{\left(S^{k-1}, s_{0}\right)}$ $=\partial_{k}\left(a_{\lambda}^{n}\right)$ and $\partial_{k} \equiv \partial_{k}\left(X_{\lambda}, X_{0 \lambda}, x_{0 \lambda}\right): \pi_{k}\left(X_{\lambda}, X_{0 \lambda}, x_{0 \lambda}\right) \rightarrow \pi_{k-1}\left(X_{0 \lambda}, x_{0 \lambda}\right)$ is a homomorphism, for all $n \in \mathbb{N}$ and $\lambda \in \Lambda$, it follows that, for all $A^{*}, B^{*} \in$ $\check{\pi}_{k}^{*}\left(X, X_{0}, x_{0}\right)$, by $(3.1)$,

$$
\begin{aligned}
\partial_{k}^{*}\left(A^{*}\right. & \left.+B^{*}\right)=\partial_{k}^{*}\left(\left\langle a^{*}\right\rangle+\left\langle b^{*}\right\rangle\right)=\partial_{k}^{*}\left\langle a^{*}+b^{*}\right\rangle=\partial_{k}^{*}\left\langle\left[\left(a_{\lambda}^{n}\right)\right]+\left[\left(b_{\lambda}^{n}\right)\right]\right\rangle \\
& =\partial_{k}^{*}\left\langle\left[\left(a_{\lambda}^{n}+b_{\lambda}^{n}\right)\right]\right\rangle=\left\langle\left[\left(\left.\left(a_{\lambda}^{n}+b_{\lambda}^{n}\right)\right|_{\left(S^{k-1}, s_{0}\right)}\right)\right]\right\rangle=\left\langle\left[\left(\partial_{k}\left(a_{\lambda}^{n}+b_{\lambda}^{n}\right)\right)\right]\right\rangle \\
& =\left\langle\left[\left(\partial_{k}\left(a_{\lambda}^{n}\right)+\partial_{k}\left(b_{\lambda}^{n}\right)\right)\right]\right\rangle=\left\langle\left[\left(\partial_{k}\left(a_{\lambda}^{n}\right)\right)\right]\right\rangle+\left\langle\left[\left(\partial_{k}\left(b_{\lambda}^{n}\right)\right)\right]\right\rangle \\
& =\left\langle\left[\left(\left.a_{\lambda}^{n}\right|_{\left(S^{k-1}, s_{0}\right)}\right)\right]\right\rangle+\left\langle\left[\left(\left.b_{\lambda}^{n}\right|_{\left(S^{k-1}, s_{0}\right)}\right)\right]\right\rangle \\
& =\left\langle\left. a^{*}\right|_{\left(S^{k-1}, s_{0}\right)}\right\rangle+\left\langle\left. b^{*}\right|_{\left(S^{k-1}, s_{0}\right)}\right\rangle=\left.A^{*}\right|_{\left(S^{k-1}, s_{0}\right)}+\left.B^{*}\right|_{\left(S^{k-1}, s_{0}\right)} \\
& =\partial_{k}^{*}\left(A^{*}\right)+\partial_{k}^{*}\left(B^{*}\right) .
\end{aligned}
$$

By that we have proven the following theorem:

THEOREM 3.4. The boundary homomorphism of the coarse shape groups $\partial_{k}^{*}$ is a group homomorphism (for $k=1$, a base point preserving function).

TheOREM 3.5. Let $X_{0}$ be normally embedded in a space $X$ and let $Y_{0}$ be normally embedded in a space $Y$. If $F^{*}:\left(X, X_{0}, x_{0}\right) \rightarrow\left(Y, Y_{0}, y_{0}\right)$ is a coarse shape morphism of $S h_{\star}^{* 2}$, then the following diagram in the Grp (Set $\left.t_{\star}\right)$ commutes for every $k \in \mathbb{N}$ :

$$
\begin{array}{lll}
\check{\pi}_{k}^{*}\left(X, X_{0}, x_{0}\right) & \stackrel{\partial_{k}^{*}}{\longrightarrow} & \check{\pi}_{k-1}^{*}\left(X_{0}, x_{0}\right) \\
\downarrow \check{\pi}_{k}^{*}\left(F^{*}\right) & & \downarrow \check{\pi}_{k-1}^{*}\left(\left.F^{*}\right|_{X_{0}}\right) . \\
\check{\pi}_{k}^{*}\left(Y, Y_{0}, y_{0}\right) & \stackrel{\partial_{k}^{*}}{\longrightarrow} & \check{\pi}_{k-1}^{*}\left(Y_{0}, y_{0}\right)
\end{array}
$$

Proof. Let $F^{*}$ be represented by $f^{*}=\left[\left(f, f_{\mu}^{n}\right)\right]:\left(X, X_{0}, x_{0}\right) \rightarrow$ $\left(Y, Y_{0}, y_{0}\right)$. For every $A^{*} \in \check{\pi}_{k}^{*}\left(X, X_{0}, x_{0}\right)$,

$$
A^{*}=\left\langle\left[\left(a_{\lambda}^{n}\right)\right]\right\rangle:\left(D^{k}, S^{k-1}, s_{0}\right) \rightarrow\left(X, X_{0}, x_{0}\right),
$$

it holds that

$$
\begin{aligned}
\partial_{k}^{*}\left(\check{\pi}_{k}^{*}\left(F^{*}\right)\left(A^{*}\right)\right) & =\partial_{k}^{*}\left(F^{*} A^{*}\right)=\left.\left(F^{*} A^{*}\right)\right|_{\left(S^{k-1}, s_{0}\right)}=\left\langle\left[\left(\left.f_{\mu}^{n} a_{f(\mu)}^{n}\right|_{\left(S^{k-1}, s_{0}\right)}\right)\right]\right\rangle \\
& =\left\langle\left[\left(\left.\left.f_{\mu}^{n}\right|_{X_{0}} \circ a_{f(\mu)}^{n}\right|_{\left(S^{k-1}, s_{0}\right)}\right)\right]\right\rangle \\
& =\left\langle\left[\left(\left.f_{\mu}^{n}\right|_{X_{0}}\right)\right]\right\rangle \circ\left\langle\left[\left(\left.a_{f(\mu)}^{n}\right|_{\left(S^{k-1}, s_{0}\right)}\right)\right]\right\rangle \\
& =\left.\left.F^{*}\right|_{X_{0}} \circ A^{*}\right|_{\left(S^{k-1}, s_{0}\right)}=\check{\pi}_{k-1}^{*}\left(\left.F^{*}\right|_{X_{0}}\right)\left(\left.A^{*}\right|_{\left(S^{k-1}, s_{0}\right)}\right) \\
& =\check{\pi}_{k-1}^{*}\left(\left.F^{*}\right|_{X_{0}}\right)\left(\partial_{k}^{*}\left(A^{*}\right)\right),
\end{aligned}
$$

which verifies commutativity of diagram (3.4).

The previous theorems imply that, for every $k \in \mathbb{N}$, the boundary homomorphism of the coarse shape groups induces a natural transformation $\partial_{k}: U_{k} \rightsquigarrow V_{k}$ of the functor $U_{k}: H T o p_{\star}^{2} \rightarrow G r p$ to the functor $V_{k}$ : 
$H T o p_{\star}^{2} \rightarrow G r p$, where $U_{k}\left(X, X_{0}, x_{0}\right)=\check{\pi}_{k}^{*}\left(X, X_{0}, x_{0}\right)$ and $V_{k}\left(X, X_{0}, x_{0}\right)=$ $\check{\pi}_{k-1}^{*}\left(X_{0}, x_{0}\right)$, for every pointed pair $\left(X, X_{0}, x_{0}\right)$ (for $k=1$, instead of $G r p$ comes $\left.S e t_{\star}\right)$

\section{The coarse shape group Sequence of a pointed pair $\left(X, X_{0}, x_{0}\right)$}

Let $S$ denote the standard pointed shape functor $S_{\left(H T o p_{\star}, H P o l_{\star}\right)}: H T o p_{\star}$ $\rightarrow S h_{\star}$ to the pointed shape category $S h_{\star}$ as well as the shape functor $S_{\left(H T o p_{\star}^{2}, H P o l_{\star}^{2}\right)}: H T o p_{\star}^{2} \rightarrow S h_{\star}^{2}$ to the pointed shape category of pairs $S h_{\star}^{2}$ (see [5, I.2.3, I.4.3]). Recall that, by general theory ([5, I.2.3]), for every $H$ mapping $f:\left(X, X_{0}, x_{0}\right) \rightarrow\left(Y, Y_{0}, y_{0}\right)$ and every pair of $H P_{o l} l_{\star}^{2}$-expansions $p$ and $q$ of $\left(X, X_{0}, x_{0}\right)$ and $\left(Y, Y_{0}, y_{0}\right)$ respectively, there exists a unique morphism $f:\left(X, X_{0}, x_{0}\right) \rightarrow\left(Y, Y_{0}, y_{0}\right)$ of pro-HPol ${ }_{\star}^{2}$ such that $f p=q\lfloor f\rfloor$ where $\lfloor f\rfloor$ denotes the rudimentary embedding of $f$ into pro-HTop $p_{\star}^{2}$. Then $f$ represents the shape morphism $F \equiv S(f):\left(X, X_{0}, x_{0}\right) \rightarrow\left(Y, Y_{0}, y_{0}\right)$ of $S h_{\star}^{2}$. Analogously, for every $H$-mapping $f:\left(X, x_{0}\right) \rightarrow\left(Y, y_{0}\right)$ and every pair of $H$ Pol $_{\star}$-expansions $p$ and $q$ of $\left(X, x_{0}\right)$ and $\left(Y, y_{0}\right)$ respectively, there exists a unique morphism $f:\left(X, x_{0}\right) \rightarrow\left(Y, y_{0}\right)$ of pro- $H P$ ol $_{\star}$ such that $f p=q\lfloor f\rfloor$. Then $f$ represents the shape morphism $F \equiv S(f):\left(X, x_{0}\right) \rightarrow\left(Y, y_{0}\right)$ of $S h_{\star}$.

Let $X_{0}$ be a subspace normally embedded in a space $X$ and let $p=\left(p_{\lambda}\right)$ : $\left(X, X_{0}, x_{0}\right) \rightarrow\left(X, X_{0}, x_{0}\right)=\left(\left(X_{\lambda}, X_{0 \lambda}, x_{0 \lambda}\right), p_{\lambda \lambda^{\prime}}, \Lambda\right)$ be a normal $H P^{\prime} l_{\star^{-}}^{2}$ expansion of the pointed pair $\left(X, X_{0}, x_{0}\right)$. If $\iota:\left(X_{0}, x_{0}\right) \rightarrow\left(X, x_{0}\right)$ and $j:\left(X,\left\{x_{0}\right\}, x_{0}\right) \rightarrow\left(X, X_{0}, x_{0}\right)$ are the homotopy classes of the inclusions, then it is easy to prove that the coarse shape morphisms $J(S(\iota))$ and $J(S(j))$ are represented by morphisms

$$
\left[\left(1_{\Lambda}, \iota_{\lambda}^{n}\right)\right]:\left(X_{0}, x_{0}\right) \rightarrow\left(X, x_{0}\right)
$$

and

$$
\left[\left(1_{\Lambda}, j_{\lambda}^{n}\right)\right]:\left(X, x_{0}, x_{0}\right) \rightarrow\left(X, X_{0}, x_{0}\right)
$$

of pro*-HPol $l_{\star}$ and pro $^{*}-H$ Pol $_{\star}^{2}$ respectively. Here $\left(1_{\Lambda}, \iota_{\lambda}^{n}\right)$ and $\left(1_{\Lambda}, j_{\lambda}^{n}\right)$ denote the level $S^{*}$-morphisms, where $\iota_{\lambda}^{n}=\iota_{\lambda}:\left(X_{0 \lambda}, x_{0 \lambda}\right) \rightarrow\left(X_{\lambda}, x_{0 \lambda}\right)$ and $j_{\lambda}^{n}=j_{\lambda}$ : $\left(X_{\lambda},\left\{x_{0 \lambda}\right\}, x_{0 \lambda}\right) \rightarrow\left(X_{\lambda}, X_{0 \lambda}, x_{0 \lambda}\right)$ are the homotopy classes of the inclusions, for all $\lambda \in \Lambda$ and $n \in \mathbb{N}$.

For every pointed pair $\left(X, X_{0}, x_{0}\right)$, where $X_{0}$ is normally embedded in $X$, one consider the following sequence:

$$
\begin{aligned}
& \ldots \stackrel{\partial_{k+1}^{*}}{\rightarrow} \check{\pi}_{k}^{*}\left(X_{0}, x_{0}\right) \stackrel{\check{I}_{k}^{*}}{\rightarrow} \check{\pi}_{k}^{*}\left(X, x_{0}\right) \stackrel{\breve{J}_{\rightarrow}^{*}}{\rightarrow} \check{\pi}_{k}^{*}\left(X, X_{0}, x_{0}\right) \stackrel{\partial_{k}^{*}}{\rightarrow} \ldots \\
& \ldots \stackrel{\check{I}_{\rightarrow}^{*}}{\rightarrow} \check{\pi}_{1}^{*}\left(X, x_{0}\right) \stackrel{\breve{J}_{\rightarrow}^{*}}{\rightarrow} \check{\pi}_{1}^{*}\left(X, X_{0}, x_{0}\right) \stackrel{\partial^{*}}{\rightarrow} \check{\pi}_{0}^{*}\left(X_{0}, x_{0}\right) \stackrel{\check{I}_{a}^{*}}{\rightarrow} \check{\pi}_{0}^{*}\left(X, x_{0}\right)
\end{aligned}
$$

which is called the coarse shape group sequence of a pointed pair $\left(X, X_{0}, x_{0}\right)$, where $\check{I}_{k}^{*}=\check{\pi}_{k}^{*}(J(S(\iota))): \check{\pi}_{k}^{*}\left(X_{0}, x_{0}\right) \rightarrow \check{\pi}_{k}^{*}\left(X, x_{0}\right)$ and $\check{J}_{k}^{*}=\check{\pi}_{k}^{*}(J(S(j)))$ : $\tilde{\pi}_{k}^{*}\left(X,\left\{x_{0}\right\}, x_{0}\right) \rightarrow \check{\pi}_{k}^{*}\left(X, X_{0}, x_{0}\right)$, for every $k \in \mathbb{N}_{0}$. 
Proposition 4.1. If a subspace $X_{0}$ is normally embedded in a space $X$, then the coarse shape group sequence of the pointed pair $\left(X, X_{0}, x_{0}\right)$ is a chain.

Proof. Let

$$
p=\left(p_{\lambda}\right):\left(X, X_{0}, x_{0}\right) \rightarrow\left(X, X_{0}, x_{0}\right)=\left(\left(X_{\lambda}, X_{0 \lambda}, x_{0 \lambda}\right), p_{\lambda \lambda^{\prime}}, \Lambda\right)
$$

be a normal $\mathrm{HPol}_{\star}^{2}$-expansion of the pointed pair $\left(X, X_{0}, x_{0}\right)$. We propose to prove semiexactness of the sequence $(4.3)$ at $\check{\pi}_{k}^{*}\left(X, x_{0}\right)$. Let $B^{*}=\left\langle\left[\left(b_{\lambda}^{n}\right)\right]\right\rangle$ : $\left(S^{k}, s_{0}\right) \rightarrow\left(X_{0}, x_{0}\right)$ be an arbitrary element of $\check{\pi}_{k}^{*}\left(X_{0}, x_{0}\right)$. By (4.1), (4.2) and (3.2) it follows that

$$
\check{J}_{k}^{*} \circ \check{I}_{k}^{*}\left(B^{*}\right)=\left\langle\left[\left(1_{\Lambda}, j_{\lambda}^{n}\right)\right]\right\rangle \circ\left\langle\left[\left(1_{\Lambda}, \iota_{\lambda}^{n}\right)\right]\right\rangle \circ\left\langle\left[\left(b_{\lambda}^{n}\right)\right]\right\rangle=\left\langle\left[\left(j_{\lambda}^{n} \circ \iota_{\lambda}^{n} \circ b_{\lambda}^{n}\right)\right]\right\rangle .
$$

By Proposition 3.2, this implies $j_{i}^{n} \circ \iota_{i}^{n} \circ b_{i}^{n}=o_{\lambda}^{n}$, for all $i, n \in \mathbb{N}$, where $o_{\lambda}^{n}$ : $\left(D^{k}, S^{k-1}, s_{0}\right) \rightarrow\left(X_{\lambda}, X_{0 \lambda}, x_{0 \lambda}\right)$ denotes the neutral element of $\pi_{k}\left(X_{\lambda}, X_{0 \lambda}\right.$, $\left.x_{0 \lambda}\right)$. Now we infer that

$$
\check{J}_{k}^{*} \circ \check{I}_{k}^{*}\left(B^{*}\right)=O^{*}=\left\langle\left[\left(o_{\lambda}^{n}\right)\right]\right\rangle .
$$

Therefore the composition $\check{J}_{k}^{*} \circ \check{I}_{k}^{*}$ is the null homomorphism and consequently $\operatorname{Im} \check{I}_{k}^{*} \subseteq \operatorname{ker} \check{J}_{k}^{*}$.

Using Proposition 3.2 and Remark 3.3, and performing obvious changes in the proof above one can analogously verify semiexactness of the sequence (4.3) at any other term.

THEOREM 4.2. Let $X_{0}$ be a subspace normally embedded in a space $X$. If the pointed pair $\left(X, X_{0}, x_{0}\right)$ admits a countable normal HPol ${ }_{\star}^{2}$-expansion, then the coarse shape group sequence of the pointed pair $\left(X, X_{0}, x_{0}\right)$ is exact.

Proof. According to $\left[6\right.$, Lemma 9], we may assume that $\left(X, X_{0}, x_{0}\right)$ admits a "sequential" normal $H P$ Pol ${ }_{\star}^{2}$-expansion $p=\left(p_{\lambda}\right):\left(X, X_{0}, x_{0}\right) \rightarrow$ $\left(X, X_{0}, x_{0}\right)=\left(\left(X_{i}, X_{0 i}, x_{0 i}\right), p_{i i+1}, \mathbb{N}\right)$. Let us prove the exactness of the sequence $(4.3)$ at $\check{\pi}_{k}^{*}\left(X, x_{0}\right)$. Referring to Proposition 4.1 , it is sufficient to prove that

$$
\operatorname{ker} \check{J}_{k}^{*} \subseteq \operatorname{Im} \check{I}_{k}^{*} \text {. }
$$

Assume that $\check{J}_{k}^{*}\left(A^{*}\right)=O^{*}$ for an $A^{*}=\left\langle\left[\left(a_{i}^{n}\right)\right]\right\rangle \in \check{\pi}_{k}^{*}\left(X, x_{0}\right)$. By $(3.2)$ and (4.2) it follows that

$$
O^{*}=\left\langle\left[\left(o_{i}^{n}\right)\right]\right\rangle=\check{J}_{k}^{*}\left(A^{*}\right)=\left\langle\left[\left(1_{\mathbb{N}}, j_{i}^{n}\right)\right]\right\rangle \circ\left\langle\left[\left(a_{i}^{n}\right)\right]\right\rangle=\left\langle\left[\left(j_{i}^{n} \circ a_{i}^{n}\right)\right]\right\rangle .
$$

Hence, for every $i \in \mathbb{N}$, there is an $n_{i} \in \mathbb{N}$ such that

$$
j_{i}^{n} \circ a_{i}^{n}=o_{i}^{n},
$$

for every $n \geqslant n_{i}$. Notice that, for all $i, n \in \mathbb{N}, a_{i}^{n}:\left(S^{k}, s_{0}\right) \rightarrow\left(X_{i}, x_{0 i}\right)$ can be regarded as an $H$-map $a_{i}^{n}:\left(D^{k}, S^{k-1}, s_{0}\right) \rightarrow\left(X_{i},\left\{x_{0 i}\right\}, x_{0 i}\right)$. Since $\left(a_{i}^{n}\right)$ is an $S^{*}$-morphism, for every $i \in \mathbb{N}$, there exists $m_{i} \in \mathbb{N}$ such that

$$
a_{j}^{n}=p_{j i} a_{i}^{n},
$$


for every $n \geqslant m_{i}$ and $j \leq i$. By (4.5) and Proposition 3.2 we infer that, for every $i \in \mathbb{N}$ and $n \geqslant n_{i}$, there exists an $H$-map $\beta_{i}^{n}:\left(S^{k}, s_{0}\right) \rightarrow\left(X_{0 i}, x_{0 i}\right)$ satisfying

$$
\iota_{i}^{n} \beta_{i}^{n}=a_{i}^{n} .
$$

In order to obtain a coarse shape morphism $B^{*} \in \check{\pi}_{k}^{*}\left(X_{0}, x_{0}\right)$ having property

$$
\check{I}_{k}^{*}\left(B^{*}\right)=A^{*}
$$

we first construct a strictly increasing sequence $\left(k_{i}\right)_{i \in \mathbb{N}_{0}}$ in $\mathbb{N}_{0}$ such that $k_{0}=0$ and $k_{i}>\max \left\{n_{i}, m_{i}\right\}$, for every $i \in \mathbb{N}$. Now, for every $n \in \mathbb{N}$, there exists a unique $i^{\prime}=i^{\prime}(n) \in \mathbb{N}_{0}$ such that $k_{i^{\prime}} \leq n<k_{i^{\prime}+1}$. Letting, for all $i, n \in \mathbb{N}$,

$$
b_{i}^{n}=\left\{\begin{array}{c}
\left.p_{i i^{\prime}}\right|_{X_{0 i^{\prime}}} \circ \beta_{i^{\prime}}^{n}, i^{\prime}=i^{\prime}(n) \geqslant i \\
o, i^{\prime}<i
\end{array}\right.
$$

we have defined an $S^{*}$-morphism $\left(b_{i}^{n}\right):\left(S^{k}, s_{0}\right) \rightarrow\left(X_{0}, x_{0}\right)$. Indeed, for every $i \in \mathbb{N}$, and every $n \geqslant k_{i}$, it holds $b_{j}^{n}=p_{j i} b_{i}^{n}$, for every $j \leq i$. It remains to verify (4.7) for $B^{*}=\left\langle\left[\left(b_{i}^{n}\right)\right]\right\rangle$. Since

$$
\check{I}_{k}^{*}\left(B^{*}\right)=\left\langle\left[\left(1_{\mathbb{N}}, \iota_{i}^{n}\right)\right]\right\rangle \circ\left\langle\left[\left(b_{i}^{n}\right)\right]\right\rangle=\left\langle\left[\left(\iota_{i}^{n} \circ b_{i}^{n}\right)\right]\right\rangle,
$$

it is sufficient to prove that $\left(\iota_{i}^{n} \circ b_{i}^{n}\right) \sim\left(a_{i}^{n}\right)$. Let $i \in \mathbb{N}$ and $n \geqslant k_{i}$ be given and let $i^{\prime}=i^{\prime}(n) \in \mathbb{N}_{0}$ be an integer such that $k_{i^{\prime}} \leq n<k_{i^{\prime}+1}$. Since, $n \geqslant k_{i^{\prime}}>\max \left\{m_{i^{\prime}}, n_{i^{\prime}}\right\}$, by combining (4.5) and (4.6), we infer that

$$
\iota_{i}^{n} \circ b_{i}^{n}=\left.\iota_{i}^{n} \circ p_{i i^{\prime}}\right|_{X_{0 i^{\prime}}} \circ \beta_{i^{\prime}}^{n}=p_{i i^{\prime}} \iota_{i^{\prime}}^{n} \beta_{i^{\prime}}^{n}=p_{i i^{\prime}} a_{i^{\prime}}^{n}=a_{i}^{n},
$$

which yields (4.7) and, consequently, (4.4). Therefore, we have proved that the sequence $(4.3)$ is exact at $\check{\pi}_{k}^{*}\left(X, x_{0}\right)$.

One can easily recognize that this proof allows straightforward translation to any other term.

Now, an immediate consequence of Theorem 2.1 and Theorem 4.2 is the following fact, which does not hold for shape groups.

Corollary 4.3. Given a closed subspace $X_{0}$ of a metric compact $X$ and an $x_{0} \in X_{0}$, then the coarse shape group sequence of the pointed pair $\left(X, X_{0}, x_{0}\right)$ is exact.

Exactness of the sequence of the coarse shape groups is very useful property for computing coarse shape groups of some particular pointed pairs of metric compacta as demonstrated by the following corollaries which easily follow from Corollary 4.3.

Corollary 4.4. Let $\left(X, X_{0}, x_{0}\right)$ be a pointed pair of metric compacta such that $\check{\pi}_{k}^{*}\left(X_{0}, x_{0}\right)=0=\check{\pi}_{k-1}^{*}\left(X_{0}, x_{0}\right)$, for some $k \in \mathbb{N}$. If $k>1$, then $\check{J}_{k}^{*}: \check{\pi}_{k}^{*}\left(X, x_{0}\right) \rightarrow \check{\pi}_{k}^{*}\left(X, X_{0}, x_{0}\right)$ is a group isomorphism. If $k=1$, then $\check{J}_{1}^{*}$ is a surjective base point preserving function. 
Corollary 4.5. Let $\left(X, X_{0}, x_{0}\right)$ be a pointed pair of metric compacta such that $\check{\pi}_{k}^{*}\left(X, x_{0}\right)=0=\check{\pi}_{k-1}^{*}\left(X, x_{0}\right)$, for some $k \in \mathbb{N}$. If $k>1$, then $\partial_{k}^{*}: \check{\pi}_{k}^{*}\left(X, X_{0}, x_{0}\right) \rightarrow \check{\pi}_{k-1}^{*}\left(X_{0}, x_{0}\right)$ is a group isomorphism. If $k=1$, then $\partial_{1}^{*}$ is a surjective base point preserving function.

Here are some examples illustrating a method for calculating coarse shape groups of some particular pointed pairs of metric compacta, satisfying assumptions of the previous corollaries.

ExAmple 4.6. Let $\left(X, X_{0}, x_{0}\right)=\lim \left(\left(X_{i}, X_{0 i}, x_{0 i}\right), p_{i i+1}, \mathbb{N}\right)$, where $X_{i}=D^{2}=\{z \in \mathbb{C}|| z \mid \leq 1\}, X_{0 i}=S^{1}=\{z \in \mathbb{C}|| z \mid=1\}, x_{0 i}=1$, and $p_{i i+1}(z)=z^{2}$, for every $i \in \mathbb{N}$. Since every $X_{i}$ is contractible $\left(X, x_{0}\right)$ has the trivial shape type. It follows that $\check{\pi}_{k}^{*}\left(X, x_{0}\right)=0$, for every $k \in \mathbb{N}_{0}$. Obviously $\left(X_{0}, x_{0}\right)$ is the pointed dyadic solenoid. Since all $H$-maps : $\left(S^{k}, s_{0}\right) \rightarrow$ $\left(X_{0 i}, x_{0 i}\right)$ are trivial, for every $k \in \mathbb{N}_{0} \backslash\{1\}$, we infer that $\check{\pi}_{k}^{*}\left(X_{0}, x_{0}\right)$ is nontrivial only for $k=1$ (see [4, Example 2.]). Now Corollary 4.5 yields $\check{\pi}_{2}^{*}\left(X, X_{0}, x_{0}\right) \cong \check{\pi}_{1}^{*}\left(X_{0}, x_{0}\right)$ and $\check{\pi}_{k}^{*}\left(X, X_{0}, x_{0}\right)=0$, for every $k \in \mathbb{N} \backslash\{2\}$.

ExAmple 4.7. Let $\left(X, X_{0}, x_{0}\right)=\lim \left(\left(X_{i}, X_{0 i}, x_{0 i}\right), p_{i i+1}, \mathbb{N}\right)$, where $X_{i}=S^{2}=\left\{(r \cos \varphi, r \sin \varphi, t) \in \mathbb{R}^{3} \mid \sqrt{r^{2}+t^{2}}=1, r, t \in \mathbb{R}, \varphi \in[0,2 \pi\rangle\right\}$, $X_{0 i}=S^{1}=\left\{(\cos \varphi, \sin \varphi, 0) \in \mathbb{R}^{3} \mid \varphi \in[0,2 \pi\rangle\right\}, x_{0 i}=(1,0,0)$, and

$$
p_{i i+1}((r \cos \varphi, r \sin \varphi, t))=\left\{\begin{array}{c}
(r \cos 3 \varphi, r \sin 3 \varphi, t), t \neq 1,-1, \\
(0,0,1), t=1, \\
(0,0,-1), t=-1
\end{array}\right.
$$

for every $i \in \mathbb{N}$. Since $\left(X_{0}, x_{0}\right)$ is the pointed solenoid whose coarse shape groups $\check{\pi}_{k}^{*}\left(X_{0}, x_{0}\right)$, for every $k \neq 1$, vanish (see the previous example), one obtains by Corollary 4.4 the following isomorphism $\check{\pi}_{k}^{*}\left(X, X_{0}, x_{0}\right) \cong$ $\check{\pi}_{k}^{*}\left(X, x_{0}\right)$, for every $k \geqslant 3$.

According to $\left[4\right.$, Theorem 2], for every $k \in \mathbb{N}_{0}$, every pointed space $\left(X, x_{0}\right)$ admits an embedding homomorphism $\left.j \equiv J\right|_{\check{\pi}_{k}\left(X, x_{0}\right)}: \check{\pi}_{k}\left(X, x_{0}\right) \rightarrow \check{\pi}_{k}^{*}\left(X, x_{0}\right)$ of the ordinary shape group $\check{\pi}_{k}\left(X, x_{0}\right)$ into the coarse shape group $\check{\pi}_{k}^{*}\left(X, x_{0}\right)$, given by the restriction of the embedding functor $J: S h_{\star} \rightarrow S h_{\star}^{*}$ to the group $\check{\pi}_{k}\left(X, x_{0}\right)$. For every pointed pair, one can also define a function

$$
\left.j \equiv J\right|_{\check{\pi}_{k}\left(X, X_{0}, x_{0}\right)}: \check{\pi}_{k}\left(X, X_{0}, x_{0}\right) \rightarrow \check{\pi}_{k}^{*}\left(X, X_{0}, x_{0}\right)
$$

by restricting the embedding functor $J: S h_{\star}^{2} \rightarrow S h_{\star}^{* 2}$ to the group $\check{\pi}_{k}\left(X, X_{0}, x_{0}\right)$. The same arguments used in the proof of [4, Theorem 2] now establish the following result (it allows to consider the shape group $\check{\pi}_{k}\left(X, X_{0}, x_{0}\right)$ as a subgroup (subset) of the coarse shape group $\left.\check{\pi}_{k}^{*}\left(X, X_{0}, x_{0}\right)\right)$ :

THEOREM 4.8. For every pointed pair $\left(X, X_{0}, x_{0}\right)$ and every $k \in \mathbb{N}_{0}, j:$ $\check{\pi}_{k}\left(X, X_{0}, x_{0}\right) \rightarrow \check{\pi}_{k}^{*}\left(X, X_{0}, x_{0}\right)$ is the embedding homomorphism of the shape 
group $\check{\pi}_{k}\left(X, X_{0}, x_{0}\right)$ to the coarse shape group $\check{\pi}_{k}^{*}\left(X, X_{0}, x_{0}\right)$. Moreover, for every shape morphism $F:\left(X, X_{0}, x_{0}\right) \rightarrow\left(Y, Y_{0}, y_{0}\right)$, the following diagram in the category Grp (Set $)_{\star}$ commutes.

$$
\begin{array}{lll}
\check{\pi}_{k}\left(X, X_{0}, x_{0}\right) & \stackrel{j}{\hookrightarrow} & \check{\pi}_{k}^{*}\left(X, X_{0}, x_{0}\right) \\
\check{\pi}_{k}(F) \downarrow & & \downarrow_{\check{\pi}_{k}^{*}(J(F))} \\
\check{\pi}_{k}\left(Y, Y_{0}, y_{0}\right) & \stackrel{j}{\hookrightarrow} & \check{\pi}_{k}^{*}\left(Y, Y_{0}, y_{0}\right)
\end{array}
$$

Let us denote $\check{I}_{k}=\check{\pi}_{k}(S(\iota)): \check{\pi}_{k}\left(X_{0}, x_{0}\right) \rightarrow \check{\pi}_{k}\left(X, x_{0}\right)$ and $\check{J}_{k}=$ $\check{\pi}_{k}(S(j)): \check{\pi}_{k}\left(X,\left\{x_{0}\right\}, x_{0}\right) \rightarrow \check{\pi}_{k}\left(X, X_{0}, x_{0}\right)$, for every $k \in \mathbb{N}_{0}$. The previous theorem assures the commutativity of the diagram (4.8) below, consisting of the shape sequence in the first row, and of the coarse shape group sequence of a pointed pair $\left(X, X_{0}, x_{0}\right)$ in the second row, where $X_{0}$ is normally embedded in $X$.

$$
\begin{array}{cccc}
\cdots \check{\pi}_{k}\left(X_{0}, x_{0}\right) & \stackrel{\check{I}_{k}}{\rightarrow} \check{\pi}_{k}\left(X, x_{0}\right) & \stackrel{\check{J}_{k}}{\rightarrow} \check{\pi}_{k}\left(X, X_{0}, x_{0}\right) & \stackrel{\partial_{k}}{\rightarrow} \check{\pi}_{k-1}\left(X_{0}, x_{0}\right) \cdots \\
\downarrow j & \downarrow j & \downarrow j & \downarrow j \\
\cdots \check{\pi}_{k}^{*}\left(X_{0}, x_{0}\right) & \stackrel{\check{I}_{k}^{*}}{\rightarrow} \check{\pi}_{k}^{*}\left(X, x_{0}\right) & \stackrel{\check{J}_{k}^{*}}{\rightarrow} \check{\pi}_{k}^{*}\left(X, X_{0}, x_{0}\right) & \stackrel{\partial_{k}^{*}}{\rightarrow} \check{\pi}_{k-1}^{*}\left(X_{0}, x_{0}\right) \cdots
\end{array}
$$

Here, $\partial_{k}: \check{\pi}_{k}\left(X, X_{0}, x_{0}\right) \rightarrow \check{\pi}_{k-1}\left(X_{0}, x_{0}\right)$ denotes the boundary homomorphism of the shape groups given by restricting shape morphisms $A$ : $\left(D^{k}, S^{k-1}, s_{0}\right) \rightarrow\left(X, X_{0}, x_{0}\right)$ to $\left(S^{k-1}, s_{0}\right)$, i.e.,

$$
\partial_{k}(A)=\left.A\right|_{\left(S^{k-1}, s_{0}\right)}:\left(S^{k-1}, s_{0}\right) \rightarrow\left(X_{0}, x_{0}\right),
$$

for every $A \in \check{\pi}_{k}\left(X, X_{0}, x_{0}\right)$. For a pointed pair of metric compacta $\left(X, X_{0}, x_{0}\right)$, the first row in diagram (4.8), generally, is not exact (see the example below or [5, Example II.3.3]), while the second row is exact.

EXAMPLE 4.9. Let, for every $i \in \mathbb{N},\left(X_{i}, X_{0 i}\right)=\left(P^{2}, P^{1}\right)$ be the pair consisting of the projective 2 -space $P^{2}$ and projective 1 -space $P^{1}$, defined as the quotient space of $\left(D^{2}, S^{1}\right)=(\{z \in \mathbb{C}|| z \mid \leq 1\},\{z \in \mathbb{C}|| z \mid=1\})$ obtained by the quotient map $\rho:\left(D^{2}, S^{1}\right) \rightarrow\left(P^{2}, P^{1}\right)$ which identifies antipodal points $z$ and $-z$ of $S^{1}$. Let $f:\left(P^{2}, P^{1}\right) \rightarrow\left(P^{2}, P^{1}\right)$ be the unique map such that $\rho \circ \tilde{f}=f \circ \rho$, where $\tilde{f}:\left(D^{2}, S^{1}\right) \rightarrow\left(D^{2}, S^{1}\right) \tilde{f}(z)=z^{3}$. Consider

$$
\left(X, X_{0}, x_{0}\right)=\lim \left(\left(X_{i}, X_{0 i}, x_{0 i}\right), p_{i i+1}, \mathbb{N}\right)
$$

where $p_{i i+1}=f$ and $x_{0 i}=\rho(1)=p_{0}, i \in \mathbb{N}$. We propose to calculate 1-dimensional (coarse) shape groups of $\left(X, x_{0}\right),\left(X_{0}, x_{0}\right)$ and $\left(X, X_{0}, x_{0}\right)$. Since every map $\left(D^{1}, S^{0}, s_{0}\right) \rightarrow\left(P^{2}, P^{1}, p_{0}\right)$ is null homotopic, we infer that $\check{\pi}_{1}^{*}\left(X, X_{0}, x_{0}\right)=\check{\pi}_{1}\left(X, X_{0}, x_{0}\right)=0$. Notice that $P^{1} \cong S^{1}$ and $\left(X_{0}, x_{0}\right)$ is homeomorphic to the solenoid. Thus, $\check{\pi}_{1}^{*}\left(X_{0}, x_{0}\right) \neq 0$ and $\check{\pi}_{1}\left(X_{0}, x_{0}\right)=0$. Since $\rho: D^{2} \rightarrow P^{2}$ is a covering map and $D^{2}$ is contractible, the elements of $\pi_{1}\left(P^{2}, p_{0}\right)$ depend only on points in the fibre $\rho^{-1}\left(p_{0}\right)$, and $\pi_{1}\left(P^{2}, p_{0}\right)=\mathbb{Z}_{2}$. 
Any path $\tilde{\alpha}$ in $D^{2}$ from 1 to -1 lifts some nontrivial loop $\alpha$ at $p_{0}$ in $P^{2}$ representing $[\alpha] \in \pi_{1}\left(P^{2}, p_{0}\right),[o] \neq[\alpha]$. Any loop $\tilde{o}$ at 1 in $D^{2}$ lifts some loop at $p_{0}$ in $P^{2}$ representing neutral element $[o] \in \pi_{1}\left(P^{2}, p_{0}\right)$. Further, $[f] \circ[\alpha]=[\alpha],[f] \circ[o]=[o]$. Therefore, the only nontrivial pointed shape morphism $A:\left(S^{1}, s_{0}\right) \rightarrow\left(X, x_{0}\right)$ is the one represented by the morphism $\left[\left(\left[\alpha_{i}\right]\right)\right]:\left(S^{1}, s_{0}\right) \rightarrow\left(\left(X_{i}, x_{0 i}\right),\left[p_{i i+1}\right], \mathbb{N}\right)$ of pro-HPol $l_{\star}, \alpha_{i}=\alpha, i \in \mathbb{N}$. One can easily see that every pointed coarse shape morphism $F^{*}=\left\langle\left[\left(f_{i}^{n}\right)\right]\right\rangle$ : $\left(S^{1}, s_{0}\right) \rightarrow\left(X, x_{0}\right)$ is uniquely determined by the sequence $\left(f_{1}^{n}\right)$ consisting of the loops $[\alpha]$ and (or) $[o]$. Sequences $\left(f_{1}^{n}\right)$ and $\left(f_{1}^{\prime n}\right)$ in $\{[\alpha],[o]\}$ represent the same coarse shape morphism if and only if they agree in all but finitely many terms. It follows that $\check{\pi}_{1}^{*}\left(X, x_{0}\right) \cong \prod_{n \in \mathbb{N}} \mathbb{Z}_{2} / \bigoplus_{n \in \mathbb{N}} \mathbb{Z}_{2}$ and $\check{\pi}_{1}\left(X, x_{0}\right)=\mathbb{Z}_{2}$. Therefore, in the following diagram the first row (consisting of the shape groups) is not exact, and, refering to Corollary 4.3, the second row (consisting of the coarse shape groups) is exact.

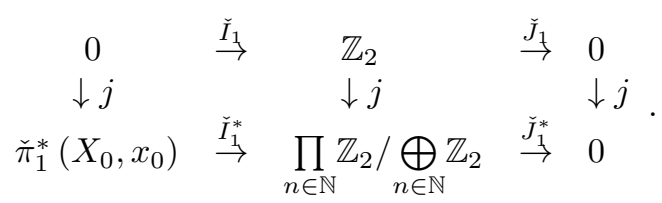

Let us clarify this situation. The exactness of the first row fails because the shape morphism $A \in \check{\pi}_{1}\left(X, x_{0}\right)$ is contained in the ker $\check{J}_{1}=\mathbb{Z}_{2}$ although it is not contained in the $\operatorname{Im} \check{I}_{1}=0$. On the other hand, the element $j(A) \in$ $\check{\pi}_{1}^{*}\left(X, x_{0}\right)$ is contained in the ker $\check{J}_{1}^{*}=\prod_{n \in \mathbb{N}} \mathbb{Z}_{2} / \bigoplus_{n \in \mathbb{N}} \mathbb{Z}_{2}$ and, by the exactness of the second row, it follows that $j(A) \in \operatorname{Im} \check{I}_{1}^{*}$. Let us find a particular coarse shape morphism $B^{*} \in \check{\pi}_{1}^{*}\left(X_{0}, x_{0}\right)$ such that $\check{I}_{1}^{*}\left(B^{*}\right)=j(A)$. The element $j(A)$ is the induced coarse shape morphism $A^{*}=J(A)$ represented by the morphism $\left[\left(\left[\alpha_{i}^{n}\right]\right)\right]:\left(S^{1}, s_{0}\right) \rightarrow\left(\left(X_{i}, x_{0 i}\right),\left[p_{i i+1}\right], \mathbb{N}\right)$ of pro $^{*}-H$ Pol $_{\star}, \alpha_{i}^{n}=\alpha$, $i, n \in \mathbb{N}$. Consider a coarse shape morphism $B^{*} \in \check{\pi}_{1}^{*}\left(X_{0}, x_{0}\right)$ represented by a morphism $\left[\left(\left[\beta_{i}^{n}\right]\right)\right]:\left(S^{1}, s_{0}\right) \rightarrow\left(\left(X_{0 i}, x_{0 i}\right),\left[\left.p_{i i+1}\right|_{X_{0 i+1}}\right], \mathbb{N}\right)$ of pro ${ }^{*}-H_{\text {Pol }}$, $\left[\beta_{i}^{n}\right]:\left(S^{1}, s_{0}\right) \rightarrow\left(X_{0 i}, x_{0 i}\right)$,

$$
\left[\beta_{i}^{n}\right]=\left\{\begin{array}{c}
{\left[\left.p_{i n}\right|_{\left.X_{0 n}\right]}\right]\left[b_{n}\right], i \leq n,} \\
{[o], i>n,}
\end{array}\right.
$$

where $\left[b_{n}\right]:\left(S^{1}, s_{0}\right) \rightarrow\left(P^{1}, p_{0}\right)$ is any group generator (or any group element having odd degree) element of $\pi_{1}\left(P^{1}, p_{0}\right)=\mathbb{Z}$. Notice that $b_{n}$ can be lifted to an arc $\tilde{b}_{n}$ in $S^{1}$ with initial point 1 and with end point -1 . Now, $\check{I}_{1}^{*}\left(B^{*}\right)$ is represented by $\left[\left(\left[\iota \circ \beta_{i}^{n}\right]\right)\right]:\left(S^{1}, s_{0}\right) \rightarrow\left(\left(X_{i}, x_{0 i}\right),\left[p_{i i+1}\right], \mathbb{N}\right)$ where $\iota:\left(P^{1}, p_{0}\right) \rightarrow\left(P^{2}, p_{0}\right)$ denotes the inclusion and $\tilde{\iota}:\left(S^{1}, 1\right) \rightarrow\left(D^{2}, 1\right)$ denotes its lifting. Hence, for an arbitrary $i \in \mathbb{N}$, it holds

$$
\left[\iota \circ \beta_{i}^{n}\right]=\left[\alpha_{i}^{n}\right] \text {, for every } n \geqslant i .
$$


Indeed, given an $n \geqslant i$, the path $\tilde{\iota} \circ f \circ \tilde{b}_{n}$ in $D^{2}$ lifts the loop $\iota \circ \beta_{i}^{n}$ in $P^{2}$, which starts at 1 and ends at -1 . Finally (4.9) implies that $\check{I}_{1}^{*}\left(B^{*}\right)=A^{*}$.

\section{REFERENCES}

[1] J. Keesling and S. Mardešić, A shape fibration with fibers of different shape, Pacific J. Math. 84 (1979), 319-331.

[2] N. Koceić Bilan and N. Uglešić, The coarse shape, Glas. Mat. Ser. III. 42(62) (2007), $145-187$.

[3] N. Koceić Bilan, On some coarse shape invariants, Topology Appl. 157 (2010), 26792685 .

[4] N. Koceić Bilan, The coarse shape groups, Topology Appl. 157 (2010), 894-901.

[5] S. Mardešić and J. Segal, Shape theory, North-Holland, North-Holland Publishing Co., Amsterdam, 1982

[6] N. Uglešić and B. Červar, The concept of a weak shape type, Int. J. Pure Appl. Math. 39 (2007), 363-428.

[7] N. Uglešić, Stability is a weak shape invariant, Glas. Mat. Ser. III 44(64) (2009), 241254

N. Koceić Bilan

Department of Mathematics, University of Split

Teslina 12/III, 21000 Split

Croatia

E-mail: koceic@pmfst.hr

Received: 17.3.2011.

Revised: 9.6.2011. 